\title{
Stability of hexaquarks in the string limit of confinement
}

\author{
J. Vijande, ${ }^{1, *}$ A. Valcarce, ${ }^{2, \dagger}$ and J.-M. Richard ${ }^{3, \dagger}$ \\ ${ }^{1}$ Departamento de Física Atómica, Molecular y Nuclear, \\ Universidad de Valencia (UV) and IFIC (UV-CSIC), Valencia, Spain. \\ ${ }^{2}$ Departamento de Física Fundamental, Universidad de Salamanca, 37008 Salamanca, Spain \\ ${ }^{3}$ Université de Lyon, Institut de Physique Nucléaire de Lyon, IN2P3-CNRS-UCBL, \\ 4 rue Enrico Fermi, 69622 Villeurbanne, France
}

(Dated: May 17, 2021)

\begin{abstract}
The stability of systems containing six quarks or antiquarks is studied within a simple string model inspired by the strong-coupling regime of quantum chromodynamics and used previously for tetraquarks and pentaquarks. We discuss both six-quark $\left(q^{6}\right)$ and three-quark-three-antiquark $\left(q^{3} \bar{q}^{3}\right)$ states. The quarks are assumed to be distinguishable and thus not submitted to antisymmetrization. It is found that the ground state of $\left(q^{6}\right)$ is stable against dissociation into two isolated baryons. For the case of $\left(q^{3} \bar{q}^{3}\right)$, our results indicate the existence of a bound state very close to the threshold. The investigations are extended to $\left(q^{3} Q^{3}\right)$ and $\left(Q^{3} \bar{q}^{3}\right)$ systems with two different constituent masses, and their stability is discussed as a function of the mass ratio.
\end{abstract}

PACS numbers: 12.39.Jh,12.40.Yx,31.15.Ar

\section{INTRODUCTION}

The situation remains unclear and even confusing in the multiquark sector. Several experimental candidates have been announced and not confirmed. Also some states with ordinary quantum numbers might be of multiquark nature or contain a large multiquark component, but their interpretation is still controversial. For a review of the experimental results, see, e.g., [1. It should be stressed, however, that the recent experimental efforts have been devoted mainly to states with hidden heavy flavor, while other sectors have never been much explored.

On the theory side, there are some uncertainties on whether the models describing ordinary mesons and baryons can be reliably extrapolated toward higher configurations, and whether these tentative models do or do not lead to stable multiquarks. In particular, the dynamics of systems made either of six quarks, $\left(q^{6}\right)$, or three quarks and three antiquarks, $\left(q^{3} \bar{q}^{3}\right)$, has been discussed by several authors, for instance 218, using nuclear forces, chromomagnetism, chiral quark models, etc.

To focus on the role of confinement, we adopt here a simple string model inspired by $[19,20$. For mesons, it reduces to a single linear potential, which can be scaled to $V_{m}=r_{12}$. For tetraquarks, the so-called "flip-flop" interaction $V_{t}=\min \left(r_{13}+r_{24}, r_{14}+r_{23}\right)$ gives binding to equal-mass configurations $(q q \bar{q} \bar{q})$ and to states with two heavy quarks and two light antiquarks $(Q Q \bar{q} \bar{q})$ [21. Stable pentaquarks are also found in an extension of this model [22]. The present article aims at studying hexaquark states, both six-quark configurations or systems made of three quarks and three antiquarks.

\footnotetext{
* javier.vijande@uv.es

$\dagger$ valcarce@usal.es

$\ddagger$ j-m.richard@ipnl.in2p3.fr
}

Our string model is extremely crude, as it neglects altogether relativistic effects, short range corrections, spindependent effects, etc. Any antisymmetrization is also disregarded, i.e., quarks are assumed of different flavors, even if bearing equal masses. There is no proliferation of multiquarks in the experimental hadron spectrum, and antisymmetrization is certainly rather effective in setting selection rules. However, before starting any detailed calculation with a refined potential and a full account of Fermi statistics, we wish to identify whether an improved picture of confinement favors the occurrence of stable multiquarks.

In early multiquark calculations, indeed, the interquark potential was taken from the naive ansatz of additive terms with color factors. Later, the flip-flop model was adopted and inserted in actual few-body calculations. The good surprise in the tetraquark and pentaquark cases [21, 22] is that the flip-flop model gives more attraction than the color-additive model, and thus suggests new scenarios for multiquark binding. Moreover, this model is supported by lattice QCD [23, 24]. This is an encouragement to extend the study of stability in the six-quark sector.

This paper is organized as follows: in Sec. III we present the linear string model which is adopted. The methods to solve the six-body problem are described in Sec. III. The results are presented in Sec. IV] before some concluding remarks in Sec. V.

\section{A SIMPLE STRING MODEL}

For mesons, the potential is taken to be the quarkantiquark separation,

$$
V_{m}(1,2)=r_{12}
$$

the string tension being set to unity, to fix the energy scale. 
For a baryon $\left(q^{3}\right)=\{1,2,3\}$, the potential is the now familiar $Y$-shape potential (see, e.g., [21] for references to early papers on this approach to the baryon dynamics)

$$
V_{Y}(1,2,3)=\min _{\ell}\left(r_{\ell 1}+r_{\ell 2}+r_{\ell 3}\right)
$$

This potential can be estimated analytically by geometric considerations. If $a, b$ and $c$ denote the sides of the triangle, namely $c=r_{12}$, etc., and $\angle a$, etc., the opposite angles, the potential reads $V_{Y}(1,2,3)=b+c$ if $\angle a>2 \pi / 3$, and permutations, and in the case where no angle exceeds $2 \pi / 3$, (see, e.g., 23])

$$
\begin{aligned}
& V_{Y}(1,2,3)=\left[\frac{a^{2}+b^{2}+c^{2}+\Lambda^{1 / 2}}{2}\right]^{1 / 2}, \\
& \Lambda=3(a+b+c)(a+b-c)(a-b+c)(-a+b+c) .
\end{aligned}
$$

For tetraquarks, the potential is taken to be minimum of the flip-flop interaction and the connected double- $Y$ diagram, both shown in Fig. 1. It reads,

$$
\begin{aligned}
V_{4}(1,2,3,4)= & \min \left[r_{13}+r_{24}, r_{14}+r_{23},\right. \\
& \left.\min _{\{k, \ell\}}\left(r_{1 k}+r_{2 k}+r_{k \ell}+r_{\ell 3}+r_{\ell 4}\right)\right] .
\end{aligned}
$$

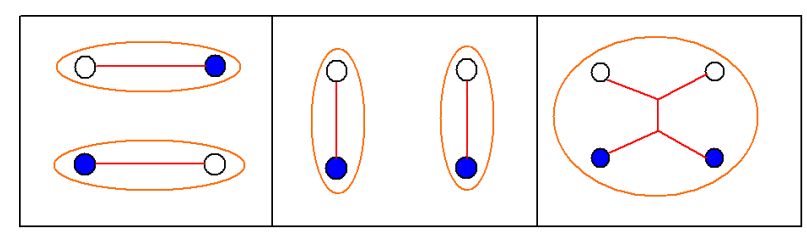

FIG. 1. Flip-flop interaction (left and center) and connected Steiner-tree diagram (right) for the tetraquark. The potential is in principle the minimum of the configurations, but it is largely dominated by the former ones.

For completeness, let us mention the pentaquark 22], though it will not enter any threshold nor sub-system in our study. The interaction is the minimum of flip-flop terms, a meson and a baryon with all permutations, and of connected Steiner trees, as shown in Fig. 2.

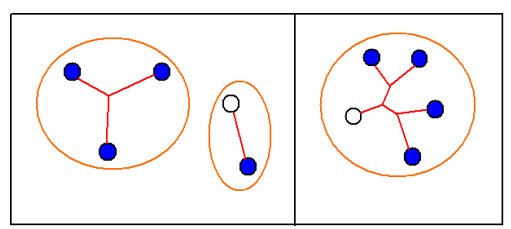

FIG. 2. Contributions to the pentaquark potential. Left: flip-flop. Right: connected Steiner tree.

For the $\left(q^{6}\right)$ configurations, there are again two types of digrams: flip-flop and connected Steiner tree, as shown in Fig. 3. The potential is minimized with respect to all permutations.

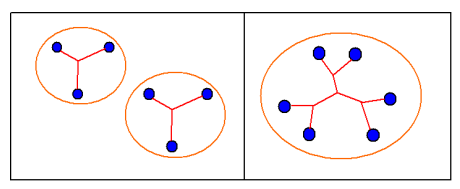

FIG. 3. Contributions to the $\left(q^{6}\right)$ potential.

Finally, for the $\left(q^{3} \bar{q}^{3}\right)$ states, there are several possibilities: flip-flop with either a baryon and an antibaryon, or three mesons, or a meson and a tetraquark, and also some connected diagrams with four or more junctions. Examples are given in Fig. 4.

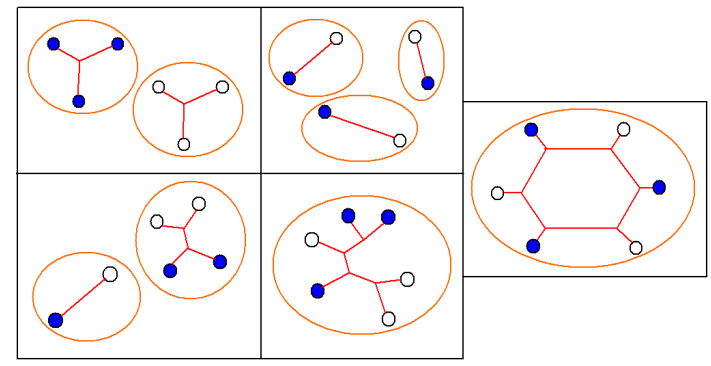

FIG. 4. Contributions to the $\left(q^{3} \bar{q}^{3}\right)$ potential.

Now, the previous studies 21 made on baryons, tetraquarks and pentaquarks have shown that the dynamics is dominated by the flip-flop terms, while the connected diagrams with $Y$-shape junctions play a minor role for binding. Moreover, the dynamics of baryon is qualitatively similar with a pair-wise potential $\sum r_{i j} / 2$ and the $Y$-shape model ${ }^{1}$

Hence for the ease of the computations, we adopt from now on the following simplified interaction:

- mesons: Eq. (1),

- baryons: the $\Delta$ interaction,

$$
V_{\Delta}(1,2,3)=\frac{1}{2}\left(r_{12}+r_{23}+r_{31}\right)
$$

- tetraquarks: the flip-flop terms,

$$
V_{t}=\min \left(r_{13}+r_{24}, r_{14}+r_{23}\right)
$$

- $\left(q^{6}\right)$ : flip-flop with a $\Delta$ interaction for each baryon, with suitable permutations, see Fig. 5 .

1 The main difference is that a baryon bound by the $Y$-potential is slightly heavier than with $\sum r_{i j} / 2$. In a refined calculation of $\left(Q^{3} \bar{q}^{3}\right)$ with two different masses, this would change the mass ratio at which there is a degeneracy of baryon-antibaryon vs. mesonic thresholds, and perhaps influence the stability of multiquarks in this region. 


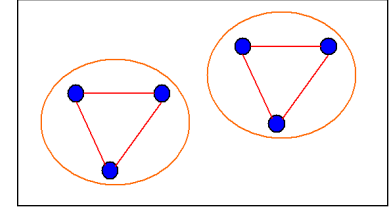

FIG. 5. Contributions to the dibaryon potential in the simplified model.

- $\left(q^{3} \bar{q}^{3}\right)$ : only the flip-flop terms, with $\Delta$ for the baryon and the antibaryon, and no double- $Y$ terms for tetraquark subsystems. See Fig. 6. The potential reads

$$
\begin{array}{r}
V_{h}(1,2,3,4,5,6)=\min [ \\
{\left[V_{\Delta}(1,2,3)+V_{\Delta}(4,5,6),\right.} \\
\left.\min _{\{i, j, k\}}\left(r_{i 4}+r_{j 5}+r_{k 6}\right)\right] .
\end{array}
$$

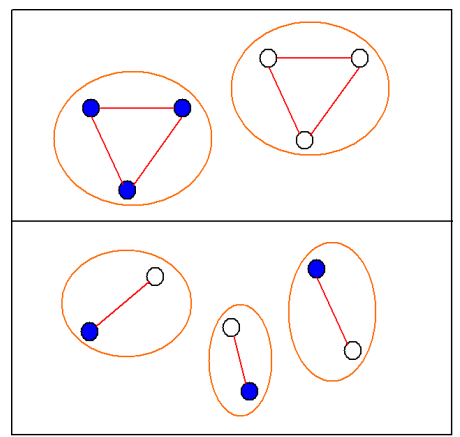

FIG. 6. Contributions to the $\left(q^{3} \bar{q}^{3}\right)$ potential in the simplified model.

\section{METHODS}

\section{A. Hyperspherical expansion}

The method of hyperspherical expansion is applicable for any set of constituent masses, but we restrict its application to the case of equal masses (but yet indistinguishable quarks). One can describe the relative motion with any standard set of Jacobi coordinates $\left\{\boldsymbol{x}_{1}, \ldots, \boldsymbol{x}_{5}\right\}$, considered as a vector in a 15-dimensional space, with spherical coordinates $(r, \Omega)$. The potential $V(r, \Omega)$ is not exactly isotropic, and the Schrödinger equation consists of an infinite set of coupled equations for the radial reduced ${ }^{2}$ partial waves $u_{L}(r)$ with generalized angular momentum $L$. A good (variational) approximation consists

${ }^{2}$ a factor $r^{7}$ is included of retaining only the $L=0$ (hyperscalar) component, which obeys $(m=\hbar=1)$

$$
-u_{0}^{\prime \prime}(r)+\frac{42}{r^{2}} u_{0}(r)+V_{00} r u_{0}(r)=E_{0} u_{0}(r),
$$

where the projection

$$
V_{00} r=\int V(r, \Omega) \mathrm{d} \Omega / \int \mathrm{d} \Omega,
$$

is computed numerically, unlike the case of a linear pairwise interaction, for which an analytic expression is available. This gives

$$
E_{0}\left(q^{6}\right)=7.230 \quad E_{0}\left(q^{3} \bar{q}^{3}\right)=7.073 .
$$

For $\left(q^{6}\right)$, the potential is fully symmetric. As in the simpler three-body problem for baryons 25, this implies that the next partial wave occurs only at $L=4$ and gives a very small correction. For $\left(q^{3} \bar{q}^{3}\right)$, there is a $L=2$ contribution. One can solve the two coupled equations that generalize (8), and get

$$
E_{2}\left(q^{3} \bar{q}^{3}\right)=6.999 .
$$

\section{B. Correlated Gaussians}

The method of expansion over Gaussians has been used for cross-check in the equal-mass case and extended to unequal constituent masses. This method is widely used in quantum chemistry and in few-body problems of nuclear physics 26, 27, with some subtle variants dealing with the most efficient manner of tuning the parameters. In our case, it reduces to a trial wave function sought as

$$
\Psi=\sum_{n=1}^{N} C_{n} \exp \left[-\sum_{i \geq j=1}^{5} a_{n i j} \boldsymbol{x}_{i} \cdot \boldsymbol{x}_{j}\right]
$$

Each individual term does not fulfill the constraints of permutation, parity, etc., but, in principle, the proper symmetry requirements are restored in the summation.

As for the Jacobi variables $\boldsymbol{x}_{i}$, a simple and universal choice, (a), consists of

$$
\begin{aligned}
& \boldsymbol{x}_{\mathbf{1}}=\boldsymbol{r}_{2}-\boldsymbol{r}_{1} \\
& \boldsymbol{x}_{\mathbf{2}}=\boldsymbol{r}_{3}-\frac{m_{1}}{m_{12}} \boldsymbol{r}_{1}-\frac{m_{2}}{m_{12}} \boldsymbol{r}_{2} \\
& \boldsymbol{x}_{\mathbf{3}}=\boldsymbol{r}_{4}-\frac{m_{1}}{m_{123}} \boldsymbol{r}_{1}-\frac{m_{2}}{m_{123}} \boldsymbol{r}_{2}-\frac{m_{3}}{m_{123}} \boldsymbol{r}_{3} \\
& \ldots \ldots
\end{aligned}
$$

where $m_{1,2, \ldots, n}=m_{1}+m_{2}+\ldots+m_{n}$, as depicted in Fig. 7) (a). As an alternative set, (b), we can choose $\boldsymbol{x}_{1}$ and $\boldsymbol{x}_{2}$ to describe the internal motion in the subset $\{1,2,3\}, \boldsymbol{x}_{4}$ and $\boldsymbol{x}_{5}$ for $\{4,5,6\}$ and $\boldsymbol{x}_{3}$ for the relative motion of the two clusters, see Fig. 7(b). This generalizes the variables used in 28 for the four-body problem. 

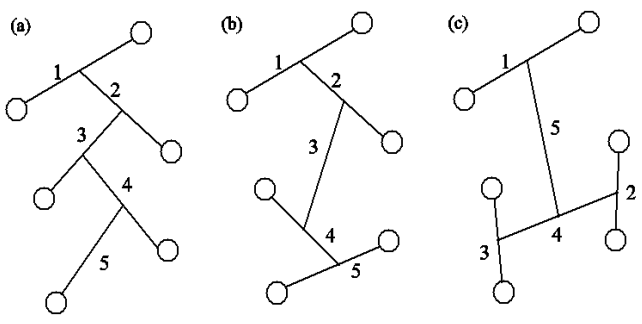

FIG. 7. Jacobi coordinates considered in this work.

We also used a set (c), where the first coordinates describe the two-body systems, and the last coordinates the relative motion of these clusters, see Fig. 7(c). In principle, the final result does not depend on the choice of Jacobi coordinates. In practice, the number of terms, $N$ in Eq. (12) is limited by the time spent in computing the matrix elements. With a finite $N$, and incomplete restoration of symmetries, the results depend on the choice of relative coordinates.

For given $a_{n i j}$, the linear parameters $C_{n}$ and the energy come from solving a generalized eigenvalue problem. Then, the non-linear parameters $a_{n i j}$ are fitted to minimize the ground-state energy. The matrix elements of the kinetic energy and normalization are known analytically. The multidimensional numerical integrals necessary to evaluate the potential matrix elements have been solved using the CUBA package 29. To guarantee the numerical accuracy of our results, several tests have been performed. As for the convergence with respect to the number $N$ of generalized Gaussians in (12), we have pushed the calculation until the energy difference obtained using $N$ and $N-1$ Gaussians became smaller than the statistical uncertainty associated with the Monte-Carlo integrations, of the order of $0.1 \%$. We have also used trial wave functions in the different set of coordinates of Fig. 7 looking for the best and fast convergence. Besides changing the Jacobi coordinates, we checked the scaling properties with respect to an overall factor applied to all masses, the virial theorem, etc. Our results are consistent within $0.05 \%$.

For a given choice of Jacobi variables, using diagonal matrices, i.e., $a_{n i j}=0$ for $i \neq j$, reduces the number of parameters. This means that the internal orbital momenta are neglected. This approximation was made in [20, where the authors used a string potential similar to ours, and led them to conclude that no six-quark bound states exist (even for the sole confinement potential, see Table I of Ref. [20]). The effect of such approximation over multiquark spectroscopy has been discussed in detail elsewhere [21, 30, 31].
TABLE I. $\left(Q^{3} q^{3}\right)$ and $\left(Q^{3} \bar{q}^{3}\right)$ thresholds, as a function of the mass ratio $M / m$, with the light quark mass set to $m=1$.

\begin{tabular}{c|ccc}
\hline \hline$M$ & $\left(q^{3}\right)+\left(Q^{3}\right)$ & $(Q \bar{q})^{3}$ & $(Q \bar{q})+\left(Q^{2} \bar{q}^{2}\right)$ \\
\hline 1 & 7.728 & 7.011 & 6.981 \\
2 & 6.929 & 6.372 & 6.335 \\
3 & 6.543 & 6.126 & 6.079 \\
4 & 6.298 & 5.997 & 5.940 \\
5 & 6.123 & 5.916 & 5.852 \\
\hline \hline
\end{tabular}

TABLE II. $\left(Q^{3} q^{3}\right)$ and $\left(Q^{3} \bar{q}^{3}\right)$ variational energies $E$, compared to their threshold energy $T$, as a function of the mass ratio $M / m$, with the light quark mass set to $m=1 . \Delta=E-T$ is the energy diffference.

\begin{tabular}{c|ccc}
\hline \hline$M$ & $E\left(Q^{3} q^{3}\right)$ & $T\left(Q^{3} q^{3}\right)$ & $\Delta\left(Q^{3} q^{3}\right)$ \\
\hline 1 & 7.237 & 7.728 & -0.491 \\
2 & 6.524 & 6.929 & -0.405 \\
3 & 6.209 & 6.543 & -0.334 \\
4 & 6.014 & 6.298 & -0.294 \\
5 & 5.890 & 6.123 & -0.233 \\
\hline$M$ & $E\left(Q^{3} \bar{q}^{3}\right)$ & $T\left(Q^{3} \bar{q}^{3}\right)$ & $\Delta\left(Q^{3} \bar{q}^{3}\right)$ \\
\hline 1 & 6.981 & 6.981 & +0.000 \\
2 & 6.314 & 6.335 & -0.021 \\
3 & 6.030 & 6.079 & -0.049 \\
4 & 5.868 & 5.940 & -0.072 \\
5 & 5.762 & 5.852 & -0.090 \\
\hline \hline
\end{tabular}

\section{RESULTS}

\section{A. Thresholds}

In Table If we compare the threshold energies for all possible decay channels. For $\left(Q^{3} q^{3}\right),(Q q q)+(Q Q q)$ is not shown, as it is always above $\left(Q^{3}\right)+\left(q^{3}\right)[25$.

For moderate values of the quark-mass ratio $M / m$, the lowest threshold of $\left(Q^{3} \bar{q}^{3}\right)$ consists of a meson plus a tetraquark state, whose energy has been calculated in Ref. 21. At higher values of $M / m$ (not shown), the lowest threshold becomes $\left(Q^{3}\right)+\left(\bar{q}^{3}\right)$.

\section{B. Hexaquark energies}

The results are shown in Table II They correspond to three terms in the Gaussian expansion (12) using either the sets (a) or (b) of coordinates.

The "dibaryon", $\left(Q^{3} q^{3}\right)$, has been studied in the range of quark-mass ratio $1 \leq M / m \leq 5$. For $M=m=1$, the result agrees quite well with the hypercentral approximation (10). The system is found stable against 
dissociation into two baryons. However, the stability deteriorates when the mass ratio increases. The behavior is reasonably linear and therefore the limit where the system becomes unbound can be estimated to be of the order of $M / m \approx 8-10$. Such a mass ratio corresponds to an intermediate value between the charm-to-light and the bottom-to-light mass ratios. Hence a triple-charm dibaryon is predicted but not a triple-beauty one. But departing from a pure linear potential would modify the value of the critical mass ratio.

In the case of $\left(Q^{3} \bar{q}^{3}\right)$, this is more delicate. For $M=m=1$, the results in Table II improve the hyperspherical estimate (11) truncated at $L=2$. It suggests that for a fully converged variational calculation, there is a shallow bound state below the lowest threshold. This means that the effective interaction between the $(q \bar{q})$ mesons is attractive. Not surprisingly for these bosonic systems, if the dimer is bound, the trimer is also bound, and a system $\left(q^{k} \bar{q}^{k}\right)$ even better for $k>3$, though the neglect of any antimmetrization becomes less and less realistic for $k>3$. Also, for $k=3$, increasing the mass $M$ in the quark sector does not modify much the effective interaction among $(q \bar{q})$ mesons, and heavier mesons experience deeper binding. To check the existence of this bound state in our model, we repeated the calculation of the equal-mass case $(M=1)$ with the set of coordinates (c). The convergence turns out much faster. We got an energy $E \simeq 6.860$ which demonstrates a deeper binding.

Now, as $M / m$ further increases, the $\left(Q^{3}\right)+\left(\bar{q}^{3}\right)$ threshold will become degenerate with the lowest mesonic threshold. This will favor binding, as the six-body wave function will contain two different decompositions into clusters with relative motion that will interfere to improve binding. However, for even larger values of the mass ratio $M / m$, no multiquark configuration can acquire enough binding to compete with the compact $\left(Q^{3}\right)$, and the system becomes unstable against rearrangement into $\left(Q^{3}\right)+\left(\bar{q}^{3}\right)$. Perhaps, some metastability could be observed with respect to some higher threshold. Similarly, models can be elaborated for hidden-charm or hidden-beauty resonances, involving four-quark configurations that are in principle unstable against $(Q \bar{Q})+(q \bar{q})$ but are mostly coupled to $(Q \bar{q})+$ c.c.

Note that the six-quark energies shown in Table II correspond to rather different wave functions. For weak binding, the wave function is mostly a hadron-hadron or a three-hadron molecule. Here, the improvements of the model could be seek as long-range nuclear forces. In the case of deep binding, we are dealing with a compact quark compound, and short-range quark forces, chromomagnetic terms, and quark antisymmetrization are required to make the estimate more realistic. To disentangle the molecular from the compact structure of these objects, one could proceed as in Ref. 28] for $\left(Q^{2} \bar{q}^{2}\right)$. Unfortunately, the necessary extension of the formalism is far beyond the scope of the present study.

We now discuss briefly the sensitivity to details of the model, restricting ourselves to the equal-mass case. We replace the perimetric $\Delta$-interaction of baryons or antibaryons is by the minima $Y$-path. For $\left(q^{6}\right)$, the threshold is now at $T=8.200$ if each baryon is estimated in the hyperscalar approximation. The same $L=0$ truncation for the six-body problem gives an energy $E=7.650$. This means that the relative amount of binding is very similar for both $\Delta$ and $Y$ cases. For $\left(q^{3} \bar{q}^{3}\right)$, this is more intricate. The threshold is not changed, as it is made of a meson and a tetraquark. However, the six-body potential is slightly increased when changing the $\Delta$ interaction by the $Y$-one, and not surprisingly, the ground-state energy also moves up, but stability is preserved.

\section{CONCLUSIONS}

Let us summarize and suggest some possible further studies.

1. The string model of confinement which combines flip-flop and connected flux tubes of minimal length gives more attraction than the additive pairwise model with color factors that was used in early multiquark calculations. The stability properties observed for some tetrraquark and pentaquark configurations is confirmed in both the six-quark and the three-quark-three-antiquark sectors.

2. This potential is flavor independent. By changing the constituent masses in the kinetic-energy part of the Hamiltonian, one can modify the binding. For tetraquarks, the message was clear: $(Q Q \bar{q} \bar{q})$ is more stable when the mass ratio $M / m$ increases, while $(Q \bar{Q} q \bar{q})$ becomes unstable. Here, the binding energy decreases for $\left(Q^{3} q^{3}\right)$, while for $\left(Q^{3} \bar{q}^{3}\right)$, it first increases and then decreases. In the limit of large $N$, no six-body configuration can compete with the deep binding of $\left(Q^{3}\right)$ that enters the lowest threshold.

3. This potential can be seen as a simplistic BornOppenheimer limit. When the quarks or antiquarks move, the gluon fluxes readjust immediately into a connected or disconnected configuration with minimal cumulated length. Thus the color part of the quark wave function is modified freely, without any antisymmetrization constraint. The model requires changes to deal with identical quarks.

4. In the $70 \mathrm{~s}$, bumps were seen in the antiproton crosssections and in the inclusive spectrum of antiproton annihilation, such as $\bar{p}+p \rightarrow \gamma X[32$, not confirmed by experiments using improved low-energy antiproton beams. Recently, some enhancements have been observed in the baryon-antibaryon mass distribution of $B$-meson or charmonium decay [33. Models have been worked out with a baryon and an antibaryon interacting by mesonic exchanges or with $\left(q^{2}-\bar{q}^{2}\right)$ quark structure [8]. The main uncertainty in the light quark sector lies in the role of annihilation. Our model, which does not include any annihilation, predicts some binding in the equal mass case. For flavor-asymmetric configurations $\left(Q^{3} \bar{q}^{3}\right)$, which are free of annihilation, the binding is improved for moder- 
ate values of the quark-mass ratio. When $Q$ becomes very large, the system breaks into a baryon and an antibaryon.

5. Dibaryon states have been often claimed but never firmly confirmed. The experimental situation remains somewhat open, as some of the most recent studies have given positive signals [34, 35. See however, [36, 37]. Our model suggests the possibility of stable dibaryon states with exotic flavor configurations. Years ago, Jaffe pointed out the possibility of coherences in the chromomagnetic interaction, and estimated that the $H$ (ssuudd) could be bound by about $150 \mathrm{MeV}$ below the $\Lambda \Lambda$ threshold. However, he used the limit of flavor SU(3) symmetry and took for (ssuudd) the short-range correlation coefficients as for ordinary baryons. Further studies indicated that $\mathrm{SU}(3)$ breaking is not favorable 3 , and that, not surprisingly, in the dilute (ssuudd), the strength of chromomagnetic effect is reduced as compared to ordinary 6. 38. However, early quark model calculations used the ansatz of pairwise interactions with color factors, $V \propto \sum \tilde{\lambda}_{i} \cdot \tilde{\lambda}_{j} v\left(r_{i j}\right)$. The string potential gives more attraction. It is interesting that two recent lattice-QCD calculations 39, 40 of the $H$ conclude to the possibility of a loosely bound or a resonance close to the threshold.

6 . The model would deserve further variational calculations, with a larger variety of constituent masses, giving the possibility of playing with the relative location of baryonic vs. mesonic thresholds.

7. Our aim is to reformulate the interaction as an operator in color space, of which the present model will be the Born-Oppenheimer limit. This, and to study the role of antisymmetrization in this new framework.

\section{ACKNOWLEDGMENTS}

This work has been partially funded by the Spanish Ministerio de Educación y Ciencia and EU FEDER under Contracts No. FPA2010-21750 and AIC10-D000503, and by the Spanish Consolider-Ingenio 2010 Program CPAN (CSD2007-00042). Some discussions with E. Hiyama, M. Oka and K. Yazaki are gratefully acknowledged.
[1] K. Nakamura et al. (Particle Data Group), J. Phys. G37, 075021 (2010).

[2] R. L. Jaffe, Phys. Rev. Lett. 38, 195 (1977), (E) 38, 617 (1977).

[3] J. L. Rosner, Phys. Rev. D33, 2043 (1986).

[4] G. Karl and P. Zenczykowski, Phys. Rev. D36, 2079 (1987).

[5] S. Fleck, C. Gignoux, J. M. Richard, and B. SilvestreBrac, Phys. Lett. B220, 616 (1989).

[6] M. Oka, Phys. Rev. D38, 298 (1988).

[7] I. S. Shapiro, Phys. Rept. 35, 129 (1978).

[8] E. Klempt, F. Bradamante, A. Martin, and J. M. Richard, Phys. Rept. 368, 119 (2002).

[9] J. T. Goldman, K. Maltman, G. J. Stephenson, Jr., K. E. Schmidt, and F. Wang, Phys. Rev. Lett. 59, 627 (1987).

[10] J. T. Goldman, K. Maltman, G. J. Stephenson, Jr., K. E. Schmidt, and F. Wang, Phys. Rev. C39, 1889 (1989).

[11] S. Takeuchi and M. Oka, Phys. Rev. Lett. 66, 1271 (1991)

[12] B. Silvestre-Brac and J. Leandri, Phys. Rev. D45, 4221 (1992).

[13] M. Cvetic, B. Golli, N. Mankoc-Borstnik, and M. Rosina, Phys. Lett. B93, 489 (1980).

[14] S. Fredriksson and M. Jändel, Phys. Rev. Lett. 48, 14 (1982).

[15] M. Anselmino, E. Predazzi, S. Ekelin, S. Fredriksson, and D. B. Lichtenberg, Rev. Mod. Phys. 65, 1199 (1993).

[16] A. Buchmann, G. Wagner, and A. Faessler, Phys. Rev. C57, 3340 (1998), arXiv:nucl-th/9803025 [nucl-th].

[17] C.-H. Chang and H.-R. Pang, Commun. Theor. Phys. 43, 275 (2005), arXiv:hep-ph/0407188

[18] J.-l. Ping, C.-r. Deng, and F. Wang, Phys. Lett. B659, 607 (2008), arXiv:hep-ph/0610390

[19] F. Lenz et al., Ann. Phys. 170, 65 (1986).
[20] J. Carlson and V. R. Pandharipande, Phys. Rev. D43, 1652 (1991).

[21] J. Vijande, A. Valcarce, and J. M. Richard, Phys. Rev. D76, 114013 (2007), arXiv:0707.3996 [hep-ph]

[22] J.-M. Richard, Phys. Rev. C81, 015205 (2010), arXiv:0908.2944 [hep-ph]

[23] T. T. Takahashi, H. Matsufuru, Y. Nemoto, and H. Suganuma, Phys. Rev. Lett. 86, 18 (2001), hep-lat/0006005.

[24] H. Suganuma, T. Iritani, F. Okiharu, T. T. Takahashi, and A. Yamamoto, AIP Conf. Proc. 1388, 195 (2011), arXiv:1103.4015 [hep-lat]

[25] J. M. Richard, Phys. Rept. 212, 1 (1992).

[26] Y. Suzuki and K. Varga, Lect. Notes Phys. M54, 1 (1998).

[27] E. Hiyama, Y. Kino, and M. Kamimura, Prog. Part. Nucl. Phys. 51, 223 (2003).

[28] J. Vijande and A. Valcarce, Phys. Rev. C80, 035204 (2009), arXiv:0908.3254 [hep-ph].

[29] T. Hahn, Computer Physics Communications 168, 78 (Jun. 2005), arXiv:hep-ph/0404043

[30] J. Vijande, F. Fernandez, A. Valcarce, and B. SilvestreBrac, Eur. Phys. J. A19, 383 (2004), arXiv:hep$\mathrm{ph} / 0310007$.

[31] J. Vijande, A. Valcarce, and N. Barnea, Phys. Rev. D79, 074010 (2009), arXiv:0903.2949 [hep-ph].

[32] L. Montanet, G. C. Rossi, and G. Veneziano, Phys. Rept. 63, 149 (1980).

[33] S.-L. Zhu, Int. J. Mod. Phys. E17, 283 (2008), arXiv:hep$\mathrm{ph} / 0703225$

[34] A. S. Khrykin et al., Phys. Rev. C64, 034002 (2001), arXiv:nucl-ex/0012011.

[35] M. Bashkanov et al., Phys. Rev. Lett. 102, 052301 (2009), arXiv:0806.4942 [nucl-ex].

[36] T. Skorodko et al., Phys. Lett. B679, 30 (2009), arXiv:0906.3087 [nucl-ex] 
[37] S. Dymov et al., Phys. Rev. Lett. 102, 192301 (2009), arXiv:0902.0715 [nucl-ex]

[38] M. Oka, K. Shimizu, and K. Yazaki, Prog. Theor. Phys. Suppl. 137, 1 (2000).

[39] T. Inoue et al. (HAL QCD), Phys. Rev. Lett. 106, 162002 (2011), arXiv:1012.5928 [hep-lat]

[40] S. R. Beane et al. (NPLQCD), Phys. Rev. Lett. 106, 162001 (2011), arXiv:1012.3812 [hep-lat] 